\title{
Dermatitis herpetiformis
}

\section{G.van Der Berg, C.A.M.RietMeyer and Th.van Joost}

Department of Dermatology and Venereology, Erasmus University and University Hospital, Rotterdam

History. A 46-year-old man suddenly developed a generalized bullous dermatosis which was diagnosed elsewhere as dermatitis herpetiformis and treated with dapsone and a gluten- and iodine-free diet. Gastroduodenoscopy at that time revealed normal features and histological examination of a duodenal biopsy specimen showed no specific changes. After a good initial response to the therapy exacerbations occurred. The lower legs and feet were very tender, but the patient never suffered from pruritus.

Examination. The face and the backs of the hands showed a moderately well defined erythema comprising multiple, taut bullae, erosions and crusts; Nikolski's sign was negative. On the lower abdomen and thighs particularly, there were irregular depigmented areas at sites where bullae had been present.

Histology and immunofluorescence studies. There was a subepidermal bulla with fibrin, erythrocytes, segmented granulocytes and nuclear debris. The superficial dermis showed a vasculitis with marked endothelial swelling with a perivascular inflammatory infiltrate and nuclear debris.

Immunofluorescence. There was a fine granular fluorescence along the basement membrane zone of IgA and this was pronounced at the tops of papillae and, to a lesser extent, in the papillary dermis. There were granular deposits of complement at the basement membrane zone, mainly at the tops of papillae.

Treatment. The dose of dapsone was increased from 50 to $100 \mathrm{mg}$ daily and despite temporary

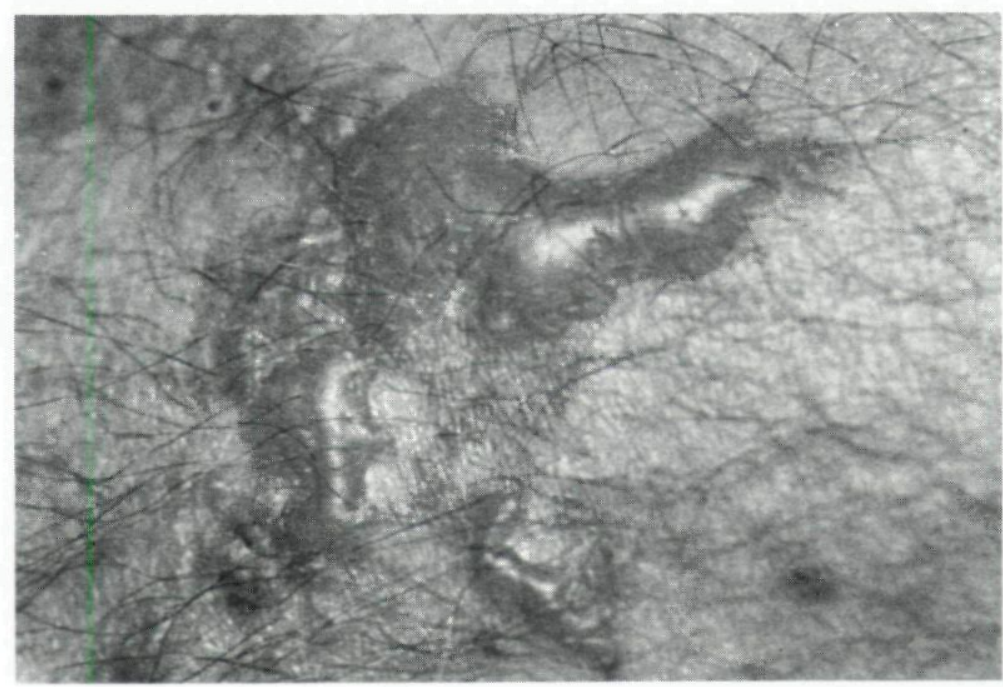

FIGURE I. Dermatitis herpetiformis with atypical manifestations. 
haemolysis there was a rapid improvement. However, the further course was characterized by remissions and exacerbations despite therapy that included a gluten- and iodine-free diet. The dapsone medication was recently supplemented with $500 \mathrm{mg}$ daily of salazosulphapyridine.

Comment. The bullous dermatosis in this patient was diagnosed as dermatitis herpetiformis on the basis of the specific immunopathological findings. However, the clinical features were not consistent with that of classical dermatitis herpetiformis. The almost normal duodenal biopsy specimen and the moderate response to diet and medication suggests another diagnosis. However, in view of the rapid recurrence after discontinuation of therapy and the repeatedly unequivocal immunopathological findings, an unusual presentation of dermatitis herpetiformis seems likely.

\section{REFERENCES}

I Meer van der JB. Granular deposits of immunoglobulins in the skin of patients with dermatitis herpetiformis: an immunofluorescent study. $\mathrm{Br}$ f Dermatol 1969; 81: 493-503.

2 Hall RP. The pathogenesis of dermatitis herpetiformis. Recent advances. 7 Am Acad Dermatol 1987; 16: 1 $129-44$.

3 Katz SI. Treatment, drugs and diet. Ann Intern Med I980; 93: 857-74.

\section{Use of the colposcope ('peniscopy') in subclinical human papilloma virus (HPV) infection of the penis}

\section{L.BLINDEMAN}

\section{Department of Dermatology and Venereology, University Hospital, Rotterdam}

History. A 27-year-old patient had for a year been the steady partner of a woman who for 2 years had condylomata acuminata of the vulva. Colposcopy revealed lesions at the level of the cervix which were histopathologically consistent with grade I cervical intraepithelial neoplasia (CIN I). Material taken from the vulval warts had yielded type I I at HPV hybridization.

The patient himself had not noticed any genital warts. Two years previously he had suffered from an epididymitis of unknown aetiology.

Examination. The external genitals showed no macroscopic lesions. Using the colposcope after application of $5 \%$ acetic acid, whitish areas were observed on the inside of the prepuce and around the external urethral orifice (Fig. I). Within the orifice a small condyloma was visible in the urethra.

Histology. (prepuce) The epidermis showed highly irregular acanthosis and slight parakeratosis. All epidermal layers contained cells showing slight-to-moderate atypia, and at these sites the normal epidermal structure was disrupted. Dyskeratotic cells were also found. The papillary dermis showed a mononuclear cellular infiltrate.

Laboratory tests. HPV typing (in situ hybridization) was negative. Urinalysis showed no 
This document is a scanned copy of a printed document. No warranty is given about the accuracy of the copy. Users should refer to the original published version of the material. 